\title{
Criteria for vaccine introduction: results of a DELPHI discussion among international immunisation experts on a stepwise decision-making procedure
}

\author{
Brigitte Piso • Ingrid Zechmeister • \\ Sabine Geiger-Gritsch
}

Received: 4 June 2010 /Accepted: 1 September 2010 /Published online: 21 September 2010

(C) Springer-Verlag 2010

\begin{abstract}
Background Based on a model of a stepwise approach for decision-making on vaccine introduction, this study aimed to reveal unpublished decision aids, to assess cut-off limits or thresholds for vaccine introduction that have already been used, and to discuss the comprehensiveness and feasibility of our suggested model.

Methods Forty international immunisation experts were invited to a DELPHI discussion, 14 finally participated. Experts received a questionnaire and were asked for comments on other experts' opinions and specification of their previously given answers in the second DELPHI round. We did not intend to develop a consensus document. Results Though most of the DELPHI participants were not aware of decision aids other than the five that had been used for the development of our model, the international discussion revealed four additional national documents that define decision-making criteria. Except for one example with a cost-utility ratio, no defined thresholds or cut-off limits have been used in vaccine introduction decisions so far. The majority of experts believe that a stepwise
\end{abstract}

B. Piso $(\square) \cdot$ I. Zechmeister $\cdot$ S. Geiger-Gritsch

Ludwig Boltzmann Institute for Health Technology Assessment,

Garnisongasse 7/20,

1090 Vienna, Austria

e-mail: brigitte.piso@hta.lbg.ac.at

URL: http://hta.lbg.ac.at

S. Geiger-Gritsch

Department of Public Health,

Information Systems and Health Technology Assessment,

UMIT University for Health Sciences,

Medical Informatics and Technology,

Eduard-Wallnöfer-Zentrum 1,

Hall in Tirol 6060, Austria approach could enhance the feasibility of decision aids. The experts agreed that the influence of each single criterion of our model should be at least "important" for decision-making. The most often mentioned possible negative consequence that could arise from a rigid stepwise procedure, was a delay of the vaccine introduction process.

Conclusions The suggested stepwise procedure provides a systematic and evidence-based standardised way to support public health immunisation policy decisions. A framework could be a common starting point.

Keywords Immunisation · Vaccination · Preventive health services

\section{Introduction}

Some of the vaccines that are currently developed or have been introduced within the last years target the prevention of less widespread, less severe and often chronic diseases at all age groups rather than severe infectious childhood diseases. Concurrently, they are more expensive than some of the even "cost-saving old vaccines". In an era of rising healthcare costs, cost-effectiveness of vaccination programmes on the population level is increasingly taken into consideration. Consecutively Health Technology Assessment (HTA) became an important tool to support decision makers as to whether or not to introduce a new health technology-including vaccines (Hutt 2008). Except for a few countries, like the UK for example, HTA based decisions are still rare and despite efforts being undertaken by the European Union, vaccination policies are moreover exclusively defined on national levels (Lopalco 2010). Therefore it is not astonishing that national immunisation 
programmes differ from country to country in their vaccination schedules and decisions regarding the implementation and funding of new vaccines.

Assuming that decisions regarding vaccine introduction should be unbiased, comprehensive and systematic and therefore be based on deliberate, rational, comprehensible and evidence-based criteria, we asked ourselves if decision aids concerning rational vaccine introduction exist at all and which criteria are crucial for a rational decision-making process. Thus, we have prepared a report assessing the availability of decision tools (Piso and Wild 2009). The comparison of the five decision aids or analytical frameworks (Stratton et al. 2000; Kimman et al. 2006; Erickson et al. 2005; World Health Organization 2005; Mansoor et al. 2000) that had been identified revealed an overall similarity with some differences in the approach as well as the criteria; the burden of disease and vaccine characteristics play a key role in all decision-making processes. Because cost-effectiveness analyses are influenced by various factors and have several limitations, views on its significance vary. Other relevant factors include the immunisation programme itself as well as its conformity with other programmes, its feasibility and how easily it can be evaluated. Additionally, acceptability, equity as well as ethical, legal and political considerations have all been mentioned, though they have been discussed to highly differing extents. Because we assumed that the most comprehensive framework possible would not provide a feasible tool for decision makers, as a result of the literature comparison, we suggested a practical, stepwise approach (Table 1) for advisers and decision makers of vaccination policy to use as a basis for vaccine introduction decisions.

The aims of this DELPHI discussion were to reveal eventually unpublished knowledge (further decision aids or introduction guidelines), to assess cut-off limits or thresholds (e.g. for costs, burden of disease or vaccine safety) that have already been used for vaccine introduction decisions in industrialised countries and to discuss our model of a stepwise decision-making approach. We did not aim at developing a consensus document.

\section{Methods}

We invited 40 international immunisation experts in 16 industrialised countries whom we had identified by contacting international HTA agencies to participate in a DELPHI discussion. Sixteen experts (in 10 countries) agreed to participate and received the first questionnaire by email. The questionnaire had been developed by two members of our institution and tested for feasibility and comprehensibility within our team. It consisted of two questions on expert's affiliation and immunisation exper- tise, two questions on identification of further decision aids, five questions on cut-off limits used in vaccine introduction decision-making and three questions to discuss our proposed stepwise decision-making model. Despite a reminder that we sent out 1 week before closure, only $50 \%$ of the experts returned their questionnaires by the end of the first deadline. Therefore the deadline was slightly expanded and participants were invited again individually. Nine weeks after the start of the first DELPHI round we extracted data of 13 completed questionnaires into an Excel sheet and SPSS database. We generated the first anonymous summary report and redistributed it to the participants for further comments. Additionally, we added three questions on applicability of DELPHI results and rational decisionmaking processes in general. Keeping in mind that the study was not aimed at a consensus document, experts were not asked to reconsider their previously given answers taking other experts' views into consideration (and answer the same questions again - in line with the commonly used DELPHI method). Experts were rather asked for comments on other experts' opinions and specification of their previously given answers wherever necessary and reasonable. Finally 12 completed questionnaires could be analysed.

\section{Results}

Participants

From 14 DELPHI participants, five work at a research institute, none work in the vaccine industry or in an international organisation (e.g. WHO). One is a national politician, five are members of national immunisation committees (affiliation and country of participants Table 2). Nine have already participated in national vaccine implementation decision-making, six in the preparation phase prior to the final decision-making and three in decisionmaking process research.

\section{Identification of further decision aids}

Most of the experts stated that they did not know of any other publications than the five previously analysed documents (Piso and Wild 2009) that are targeted at rational vaccine implementation decision-making in industrialised countries. Nevertheless three further documents could be identified by DELPHI participants: a Brazilian (Castillo-Solórzano and Andrus 2004) publication that deals with decision-making on the development of new vaccines, an Australian document (Australian Government Department of Health and Ageing Pharmaceutical Benefits Advisory Committee 2008) that aims at 
Table 1 Suggested stepwise procedure for vaccine introduction decision making

Step Criteria to be assessed ${ }^{\mathrm{a}}$

Step 1 Fundamental considerations concerning the disease as a public health problem and alternative operational and delivery strategies (alternative measures)

1. Aims of the planned immunisation strategy

2. Comparison with other programmes (conformity of programmes)

3. Availability of sufficient basic research data (research questions)

Step 2 4. "Disease" considerations, e.g.:

Burden of disease

5. "Vaccine" considerations, e.g.:

Clinical manifestations

Vaccine characteristics

Current treatment

Supply

Epidemiology

Administration schedule

Risk groups and risk factors

Immune response

Social impact

Efficacy and utilisation

Other preventive measures

Population effectiveness

Safety

Step 3 6. Cost-effectiveness analysis

Step 4 7. Considerations on acceptability

8. Feasibility of the new programme

9. Implications on equity

10. Ethical considerations

11. Legal considerations

12. Political considerations

13. Potential side effects (e.g.: vaccine side effects, feasibility side effects or utilization side effects)

Step 5 Decision making process itself (final decision)

Step 6 Implementation

Step 7 14. Surveillance

of vaccine coverage and utilisation

of epidemiologic changes, the frequency and nature of adverse events

and immune surveillance and re-evaluation (revision)

${ }^{\mathrm{a}}$ The consecutive numbers reflect the 14 criteria to be considered in a vaccine introduction decision-making process that have been identified in our previous work (Piso and Wild 2009); key criteria in bold letters

Table 2 Affiliation and country of DELPHI participants

\begin{tabular}{ll}
\hline Institution & Country \\
\hline National Centre for Immunisation Research \& Surveillance (NCIRS) & Australia \\
Australian Government, Department of Health and Ageing & Australia \\
Medical University of Vienna, Insitute of Specific Prophylaxis and Tropical Medicine & Austria \\
National immunisation committee & Austria \\
Belgian Health Care Knowledge Centre (KCE) & Belgium \\
University of São Paulo & Brazil \\
McGill University Health Centre & Canada \\
National immunisation committee & Germany \\
Ministry of Health and Consumers Affairs, DG of Public Health & Spain \\
Junta de Andalucía, Departement of Health & Spain \\
The National Board of Health and Welfare, Communicable Disease Prevention and Control & Sweden \\
NHS Greater Glasgow and Clyde & United Kingdom \\
NHS Department of Health/Immunisation & United Kingdom \\
US Department of Veterans Affairs, National Center for Health Promotion and Disease Prevention & USA \\
\hline
\end{tabular}


rational medicine implementation decision-making and a Spanish document (González Alonso et al. 2004) which points out the main criteria that can be taken into account to introduce any change in the vaccination programme. The latter criteria are related to the burden of disease, intrinsic factors of the vaccine, the cost effectiveness of the vaccine, the impact of the new vaccine for the vaccination scheme and other aspects such as acceptability as well as equity and legal considerations. Additionally, in the pre-Delphi phase, one further document had been revealed by a member of the Dutch HTA agency (Houweling et al. 2010). In this report, seven criteria for the inclusion of vaccinations in public programmes have been defined. These criteria cover the seriousness and extent of the disease burden, the effectiveness and safety of the vaccination as well as the acceptability, the efficiency (favourable cost-benefit ratio in relation to alternative measures) and the priority of the vaccination (urgency of the public health need).

The majority of experts also did not know of (un) published guidelines or institutional manuals, only one referred to the existence of "unpublished national guidelines" in her own country. The two Australian experts referred to proformas that communicate advice on decisionmaking between peak bodies: one is based on a structured set of questions agreed between the two bodies (committees) and precedes an application for funding within the national immunisation programme; the other responds to specific technical questions about the application following a preliminary review of the application. Each proforma is completed by the technical advisory committee and is considered by the recommending committee. Each completed proforma is also provided to the relevant applicant, who can comment on the information provided before any recommendation is considered.

Identification of cut-off limits or thresholds that have already been used in the decision-making process

Cost-utility threshold (\$/QALY, $€ / Q A L Y)$ Vaccines have been considered to be favourable because they showed a cost-utility ratio below a defined threshold just in one country (about 50,000 US\$ or 35,000€/QALY). One expert referred to an implied threshold (from about 30,000 US\$/ $22,000 €$ to 57,000 US\$ $\$ 40,000 €$ per life year saved), that was derived from a retrospective study which analysed the consistency of funding decisions during a given time period (George et al. 2001). Other experts did not give any defined thresholds but stated that cost-utility data were somehow considered and that thresholds may differ according to various and changing economic situations.

Domestic burden of disease The majority of experts stated that criteria related to the burden of disease have been used (e.g.: epidemiology, clinical manifestations, mortality, costs, social impact, hospitalisation, medical attendance or disease sequelae). Most of them agreed that there were neither defined "cut-off limits" nor a predefined minimal burden of disease.

Effectiveness Most experts pointed out, that no quantitative cut-off levels of proposed vaccine effectiveness have been used, "although effectiveness is considered separately as a 'building block' of the overall assessment". They explained that effectiveness is "incorporated in the cost-effectiveness model", "a relevant factor in its own right" and "is considered more in the context of overall analysis, including a sensitivity analysis". Vaccine effectiveness is also "considered in vaccine development" and a cut-off limit would "depend on the disease for which the vaccination is proposed". One expert mentioned that an efficacy less than $70 \%$ would be questionable, whereas another one stated that an efficacy of $50 \%$ may be acceptable. In the second DELPHI round, one expert pointed out, that efficacy thresholds should primarily depend on the outcome being measured (e.g. immunogenicity outcome vs. directly patient-relevant outcome).

Safety None of the experts gave a defined "safety level" but some referred to safety issues that have been considered in vaccine introduction decision-making: thus, safety is for example "taken into account in the cost effectiveness model" and "depends on the risk/benefit balance and the absence of related serious adverse events". One expert defined safety as "registered by the national medical products agency". Another expert stated in the discussion that "safety versus efficacy has a changing value with respect to the severity of the disease and that safety considerations have a much higher ranking in socioeconomically well-situated countries".

Number needed to vaccinate Except for two experts who gave examples in which the number needed to vaccinate (NNV) has already been used as decision criterion (in HPV and Herpes Zoster vaccination decisions), most experts agreed, that no defined number needed to vaccinate (NNV) has been used. They see the NNV "incorporated into the cost effectiveness model" or as "one measure of vaccine effectiveness". Still, "the interpretation of an effectiveness outcome should depend on the nature of the outcome being presented". For example, "the NNV to get an extra immunological response should be interpreted differently to the NNV to avoid one extra death". Additionally, "the NNV should always include the time frame of observation (e.g. three months vs. twenty years)". In case of "vaccines preventing noncommunical diseases (e.g. Tetanus) that provide personal protection only, each immunised individ- 
ual may benefit". By contrast, in communicable diseases herd effects have to be taken into NNV considerations: "The example of rubella vaccination for the prevention of congenital rubella syndrome has shown that a too low immunisation rate may have worse effects than no vaccination."

Discussion of the criteria to be taken into consideration and the suggested stepwise procedure

None of the DELPHI participants considered the listed criteria (Table 1) redundant. Only three experts added criteria for rational decision-making that they assumed to be missing in the synthesis of the decision support document (Piso and Wild 2009): "the impact on current disease management or prevention" and "immunological interference with other vaccines or the effect on risk behaviour that might increase risk of other diseases spread" were mentioned, although these considerations had already been covered by the side-effects criterion (see step 4 in Table 1). Also, "the need for re-evaluation of decision, especially when long-term effects are unknown at initial decision point was added, even though the last step of the decisionmaking model already emphasises the need of re-evaluation of decisions. Furthermore "the importance of uncertainties" was mentioned, though they should be considered in step 1 in the field of research questions (that have not been answered sufficiently at the time of decision-making).

The majority of participants agreed that there are no general more or less important criteria to be fulfilled or considered in the decision-making process. Others pointed out that "disease burden is most important regardless of (reasonable) costs" or in contrast, an "acceptable incremental cost-effectiveness, preferably with minimal uncertainty" is the most important decision criterion. Other major considerations mentioned were, associated risks, vaccine production capacity and any hurdles for marketing a vaccine. The latter, however, should even be discussed before large trials are initiated.

Next, participants ranked the 14 criteria, that had been identified in our decision support paper (Piso and Wild 2009), dependent on the influence these criteria should have on vaccine introduction decisions on a scale between 1 (very important) and 5 (not important). The median of grades ranged from 1 to 3 , therefore experts agreed that the influence of each single criterion should at least be "important" (Table 3). Burden of disease, vaccine, side effects and ethical considerations were considered to be most important (median 1.0).

The majority of experts argued that this ranking is dependent on the type of vaccine and the disease against it is introduced, respectively. They acknowledged that the 
"importance of criteria is variable depending on the individual case, especially the severity of the disease, whether there is already a vaccine available for the intended purpose or not" and that it is "dependant on the type of vaccine (e.g. attenuated or live)". Examples of crucial criteria that led to a decision to introduce or to withhold a vaccine from a national immunisation programme in the past were given by almost all participants, e.g.:

- The burden of disease and the increasing number of cases (meningococcal $\mathrm{C}$ vaccine)

- Epidemiology and sequalae (herpes zoster vaccine)

- High hospitalisation rate (rotavirus vaccine)

- Political considerations (human papilloma virus and herpes zoster vaccine)

- Health economic aspects (pneumococcal vaccine)

- Price negotiations due to an unfavourable incremental cost-effectiveness ratio (human papilloma virus vaccine)

- Side effects (vaccines against tuberculosis or polyomyelitis)

- A lower than expected efficacy (hexavalent vaccine against diphtheria, tetanus, pertussis, hepatitis B, poliomyelitis and Haemophilus influenzae type b) as well as

- Issues that relate to long-term effects of vaccination and the number of areas of uncertainty (herpes zoster and varicella vaccine)

The majority of experts believe that a stepwise approach could enhance the feasibility of decision aids. Most of them even stated that the order and the hierarchy of steps and the criteria themselves should not be changed or moved to a different step. While one participant suggested to change the order of steps 3 and 4, another participant disagreed, because he stated: "the reason to have a logical order was to help ensure consistent decision-making over time" and "all the influential information of steps 1-4 must be taken into consideration for the decision in step 5" anyway. "The presentation of information in a consistent order" would be "crucial to efficient decision-making in a systematic manner across a series of health care interventions". Another participant suggested, that "HTA agencies need to get involved in a step 0 , deciding together with efficacy and safety agencies on primary endpoints and criteria to reach before the starting of large phase 3 trials". In the discussion a different expert commented this suggestion as "laudable", but pointed out that also HTA agencies may want to change their position during the following years, especially "if the lead time to a return on investment is many years during which time the understanding of the disease may move on". Another expert mitigated the role of HTA agencies by stating that "HTA agencies are but one part of the decisionmaking process". Another expert highlighted "the need for re-evaluation and repetition of the stepwise evaluation as soon as data become available, especially if the results are long-term". The conclusion that "many steps are dependent on each other and cannot only be considered alone" was supported by a different participant in the discussion.

Most experts gave reasons for situations in which one or more steps have been or would likely to be skipped. "If planning is reactive rather than proactive, political interests" might have a greater influence on vaccine introduction "than evidence-based criteria". Health economic considerations might be less important if a "vaccine for a frequent short-term life-threatening disease is effective (e.g. pandemic)". The "immediacy of a problem" might be high, therefore one "may not be able to wait until all data is available". The "public health relevance of disease prevention and prevention of its consequences" might be considered "more important than the burden of disease" (for the individual). Another expert thought, that "step 1 could sometimes come after step 2 , as the availability of a new vaccine from a vaccine manufacturer has sometimes been the impetus to assess the public health burden of disease." Half of the participants thought that no negative consequences could arise from a stepwise procedure "unless the steps were too rigidly implemented" and it was not "necessary to cover one step before going to the next one". The other half mentioned possible negative consequences, most often "a delay of the vaccine introduction process". This aspect was considered "serious with respect to the everywhere growing administrative bureaucracy". One the other hand one participant totally disagreed that a stepwise procedure would lead to a delay and endorsed that it is even "a way to have a more organised process".

All experts see strengths of the proposed model. It is "complete and logical" and therefore "its comprehensiveness and thoroughness helps, that all relevant factors are appropriately considered and addressed". Its "clarity and structure may help to avoid mistakes" and it enhances "transparency". It provides a "systematic and evidence based standardised way ("a tool") to support public health policy decision." It was seen to be "easy to repeat" and therefore easy to "compare with others".

A potential weakness of the model could be that it "may need to be flexible in a public health emergency". Additionally, a stepwise decision-making tool could be "weaker than political and economic rationalities" and "too exhaustive for busy decision makers". Furthermore, there "may not be adequate data to access all factors" and "even within one healthcare system" people "may weigh the factors differently, leading to disagreements." One expert mentioned that the decision-making tool "may not accurately encompass particular characteristics of a new intervention". Therefore he stated that "a deliberative committee process" would be more 
relevant for decision makers "than applying a formulaic approach to decision-making."

\section{Discussion}

Though most of the DELPHI participants were not aware of other decision aids, the international discussion revealed four additional national documents that define decisionmaking criteria. Except of one example for a cost-utility ratio, no defined thresholds or cut-off limits have been used in vaccine introduction decisions so far. All experts agreed that the influence of each single criterion of our model should be at least "important". Almost all participants were convinced that a stepwise approach could enhance the feasibility of decision aids.

All experts acknowledged that they gained some useful information by participating in the DELPHI discussion. Participation permitted them "to know other expert opinions and other documents used in other countries" and most of them thought that the information will be applicable in vaccine implementation discussion or decision-making they are involved in and it could even be used "to improve the own national guideline for the decision-making process". Only one participant "detected nothing specific or unexpected which would be sufficient to form the basis of arguing for a change to the way they have already approached this issue in the national context".

The results of this Delphi discussion among international vaccination experts reflects that "national immunisation programmes are influenced by political and public opinion about money spending priorities and may not reflect health care priorities" and "many decisions have to be made when not all important information is available." But nevertheless experts agreed that "guidelines may be helpful" to "articulate different rationalities" and to make the decision-making process "more evidence-based than based on political considerations". "Careful consideration of available evidence (using defined criteria), current context and ongoing discussions in a network of national and international experts" would at least "help to make the best decisions regarding immunisation programme planning". But even then decisions could only be "rational for a given context at a given point in time". "Identifying some areas of uncertainty" would not "mean a decision is not rational", but the process would have to be "re-evaluated as more information is available".

Having said this, a more or less consistent structure on which vaccine implementation decisions are based on, could help all of us: vaccine industry would be supported in vaccine development and the following application procedure, if implementation criteria were transparent. Decision makers could insist on pre-defined information that has to be provided prior to the decision-making process. Last but not least, a clearly communicated decision would help health care workers as well as consumers to comprehend whether or not a vaccine has been implemented in a national programme. Consequently, sharing all available information would make decisions on both levels - the population and the individual level - more evidence-based rather than based on fear.

The increasing number of vaccines implemented in programmes on one hand and the decreasing awareness of diseases that became less common due to successful vaccination programmes on the other, already threatens programme acceptability in the population. Therefore, only a structured, comprehensible decision-making process based on transparent decision criteria will strengthen vaccination as an effective public health tool in the future.

In this DELPHI discussion, 14 experts from 10 industrialised countries, who have experience in the field of vaccine introduction decision making, gave insight to the basic principles of national decision-making and its process. We did not aim at achieving a consensus document, though we would appreciate any efforts in this direction. In our opinion, a consensus among decision makers could only be reached by discussions on a high political level, moderated by an international organisation (e.g. the WHO or the European Commission). It will not be easily achieved while decision making criteria still show a wide range between countries or are even completely untransparent. Therefore the results of this DELPHI discussion could be a starting point for such efforts.

\section{Limitations}

Because of the small number of immunisation experts participating in this Delphi discussion, the quantitative analysis of the DELPHI results is not representative for the whole immunisation community. Results should rather be discussed in a qualitative manner.

Acknowledgements First of all the authors gratefully acknowledge the time and effort of the DELPHI participants, without whom this project would not have been possible. Furthermore we thank Claudia Wild, the head of our HTA institution, for idea input and guidance as well as our colleague Stefan Mathis for the technical support in the preparation of the word form documents and the data export.

Conflict of interest The authors declare that they have no conflict of interest.

\section{References}

Australian Government Department of Health and Ageing Pharmaceutical Benefits Advisory Committee (2008) Guidelines for preparing 
submissions to the pharmaceutical benefits advisory committee (version 4.3). Commonwealth of Australia. http://www.health.gov. au/internet/main/publishing.nsf/Content/pbacguidelines-index. Cited 4 June 2010

Castillo-Solórzano C, Andrus J (2004) El desarrollo de nuevas vacunas: generación de información para la toma de decisions. Rev Panam Salud Públ 15(1):1-3

Erickson LJ, De Wals P, Farand L (2005) An analytical framework for immunization programs in Canada. Vaccine 23(19):2470-2476

George B, Harris A, Mitchell A (2001) Cost-effectiveness analysis and the consistency of decision-making: evidence from pharmaceutical reimbursement in Australia (1991 to 1996). Pharmacoeconomics 19(11):1103-1109

González Alonso J, Pachón del Amo I, Anchuela OT, Heras CA, Peña-Rey Lorenzo I, García FS, et al (2004) Criterios para fundamentar la modificación de los programas de vacunas. Ministerio de sanidad y consumo- Subdirección general de promoción de la salud y epidemiología. http://www.msc.es/ ciudadanos/proteccionSalud/vacunaciones/docs/criteriosVacunas. pdf. Cited 4 June 2010

Houweling H, Verweij M, Ruitenberg EJ (2010) Criteria for inclusion of vaccinations in public programmes. Vaccine 28 (17):2924-2931

Hutt HJ (2008) How do we tackle the complex field of vaccinology in the twenty-first century? J Public Health 16(4): 245-246
Kimman TG, Boot HJ, Berbers GAM, Vermeer-de Bondt PE, Ardine de Wit G, de Melker HE (2006) Developing a vaccination evaluation model to support evidence-based decision-making on national immunization programs. Vaccine 24(22):4769-4778

Lopalco PL (2010) Improving vaccination programmes in the European Union. J Public Health 16(4): 281-285

Mansoor O, Shin S, Maher C, and the Immunization Focus of WPRO (2000) Assessing new vaccines for national immunization programmes: a framework to assist decision makers. World Health Organization Western Pacific Regional Office. http:// whqlibdoc.who.int/hq/2000/9290611545.pdf. Cited 4 June 2010

Piso B, Wild C (2009) Decision support in vaccination policies. Vaccine 27(43):5923-5928

Stratton K, Durch J, Lawrence S, Editors Committee to Study Priorities for Vaccine Development Division of Health Promotion and Disease Prevention Institute of Medicine (2000) Vaccines for the 21st century: a tool for decisionmaking. National Academies Press, Washington, DC

World Health Organization (2005) Expanded programme on immunization of the department of immunization, vaccines and biologicals. Introduction guideline- adding a vaccine to a national immunization programme: decision and implementation. WHO. http://whqlibdoc.who.int/hq/2005/WHO_IVB_05.18.pdf. Cited 4 June 2010 